\title{
Effectiveness of the Laser on TMD - Systematic Review
}

\author{
A. Mouhibi ${ }^{1}$, A. Chafii ${ }^{2}$, A. Andoh ${ }^{3}$
}

\section{Introduction}

Temporo-Mandibular Disorders (TMD) are the symptomatic expression of amanducatory system pathology that may be of muscular or articular origin. It includes the anatomical, histological and functional abnormalities of the system. Standard treatment using occlusal splintis considered to beonerous and expensive; The new era of the Laser has allowed a great evolution of the dental practice, especialy in occlusodontics, thanks to: Low Level Laser Therapy (LLLT) which is a low energy light application allowing to increase the speed and the quality of the injured tissues healing process(fig 1). This treatment is limited to a few sessions and a few minutes.

Its mechanism of action is based on the increase of cellular energy (ATP) and the reduction of free radicals (oxidative stress).

This work is a systematic review that aims to analyze the scientificliterature during the period 2007 to 2017 for all articles dealing with the effectiveness of the Laser in disorders of the manducatory system and to answer the following issues:

* Whatis the place of the laser in the treatment of TMD?

* Is the laser an alternative to occlusal splints?

* Is the laser an indispensable tool in clinical practice?

\section{Material and Method}

\section{Material}

An exhaustive bibliographical search was carried out on the PubMed database with MeSH words and according to the PICO method.

MeShwords used: Laser therapy, lowlevel laser therapy, temporomandibular joint, temporomandibular joint disorders, temporomandibular joint dysfunction syndrome, facial pain, occlusal splint, temporomandibular disorders.

\section{Inclusion criteria:}

Articles dealingwith the effectiveness of Laser in disorders of the manducatoryapparatus; RandomizedClinical Trials (RCTs), Meta-Analysis and SystematicReviews. The selectedperiod: from 2007 to 2017

\section{Exclusion criteria:}

Animal Studies and Case Studies (Case Report)

\section{Method}

Selection of articles meeting the inclusion criteria based on their titles and abstracts, then critical reading of the retained articles full text by applying the appropriate reading grids to each type of study.

Two rearders have checked the criteria and red the articles to confirm eligibility.

We followed the metholodogy of PICO by definingthe « $\mathrm{P}$ » as TMD : the patients whom consult for TMD, «I» as the Laser, « $\mathrm{C} »$ as the occlusal splint which is the gold standard treatment and the « $\mathrm{O} »$ as efficiency regarding the pain and mandibular motility.

\section{Results}

The results of the research was as follows: $2 \mathrm{RCT}$ and 1 systematic review :

\begin{tabular}{|c|c|c|c|c|}
\hline Articls & Study Type & Sample & !? & Results \\
\hline 1 & RCT & 30 & Pain score & $\begin{array}{c}\text { Equal efficacy of } \\
\text { LLT and Occlusal } \\
\text { splint }\end{array}$ \\
\hline 2 & RCT & 9 & $\begin{array}{c}\text { Pain score }+ \\
\text { distribution of } \\
\text { occlusal } \\
\text { surfaces }\end{array}$ & $\begin{array}{c}\text { No significant } \\
\text { improvement in } \\
\text { LLT and Placebo }\end{array}$ \\
\hline 3 & $\begin{array}{c}\text { Systématic } \\
\text { revue }\end{array}$ & 14 RCT & $\begin{array}{c}\text { RDC/TMD, } \\
\text { EVA, ... }\end{array}$ & $\begin{array}{c}\text { All studies showed } \\
\text { significant } \\
\text { improvement except } \\
\text { for one study. }\end{array}$ \\
\hline
\end{tabular}

In the first study, the authors evaluated the pain with the "Pain score" tool for 3 samples of 10 patients. A different treatment was used in each group: Occlusal splint, LLT and Placebo.

The results showed an improvement for the 2 treated groups, whichmeansthat the LLT is as effective as the occlusal splint. Figure 2

For the second study, the pain and the distribution of the Occlusal surfaces were assessed using the "Pain score" and "T-Scan" tools for 2 samples of 9 patients:

5 from the Experimental group and 4 from the Placebo group. The results showed no significant improvement between the LLT and Placebo treated group.

The third study, a systematic review, evaluated several publications and concluded that there was no consensus regarding laser efficiency, and that thers's adiversity of protocols, energy level, density, application time, frequency, sessions number, and reassessment and follow-up periods. 


\section{International Journal of Science and Research (IJSR) \\ ISSN: 2319-7064}

Index Copernicus Value (2016): 79.57 | Impact Factor (2017): 7.296

\section{Discussion}

Publications on the use of LLT for TMD treatment are limited. However, the low-level laser therapy has shown equal efficiency to that of conventional treatment by occlusal splint for the treatment of muscular pathologies, and joint pain.

LLT appears to play an important role in the management of patients with TMD and the use of the laser is promising and has several advantages.

The studies selected, despite their limitations, as the small samples studied and the non-standardization of their protocols, report the lack of evidence and consensus regarding the dose administered, the duration of appliance, and the long-term effects of this treatment. The lack of standardization leads to different results and makes comparison between studies difficult.

More research should be directed towards standardization of all these factors.

\section{Conclusion}

The treatment of TMD is complex because of theirmultifactorialetiology. LLLT has been accepted as a good adjunctive therapy in pain control, stimulation of healing and improvement of functional conditions. Laser treatment remains a symptomatic treatment. Despite all the commercial advices, the new era of laser must betaken with care. Further studies are essential to reach consensus on the best appliance protocol, greater clinical experience, and evidence-based results. 